\title{
Quantitative Studie des Nebennierenmarks und -rinde beim erwachsenen Kaninchen.
}

\author{
Von \\ Takeo Onozawa. \\ Aus dem Anatomischen Institut der Keio Universität, Tokyo.
}

\section{Einleitung.}

Über das quantitative Verhältnis des Nebennierenmarks und -rinde haben viele Autoren wie Canalis (87), Hultgren und Anderson (99), Soulié (03), Scheel (08), Elliot und Tuckett (06), Starkel und Wegrzynski (10), Thomas (11), Bager (17), Jackson (19), Donaldson (19), Castaldi (22), Hammar (24), Hett $(26,28)$ u.a., besonders seit die exakte Berechnungsmethode von Elliot (06) und Hammar (10) veröffentlicht ist, eingehende Studien gemacht. Sie beschäftigten sich bei den Säugetieren und Menschen, unter Berücksichtigung des Alters, Körpergewichts und Geschlechts, mit der Verfolgung des gegenseitigen Mengenverhältnisses dieser beiden Substanzen.

Nach Elliot (06) vergrössert sich die Nebemniere bei Katze, Kaninchen und Meerschweinchen besonders schnell bis zur Pubertät und noch darüber hinaus dauert die Zunahme, während das Mark selbst nach der Pubertät im Wachstum aufhört oder sich doch nur wenig ausdehnt. Auch andere Forscher wie Canalis (87), Hultgren und Anderson (99), Donaldson (19), Jackson (19). Castaldi (22) u.a. wiesen bei den Säugetieren auf die Abnahme des relativen Volumens des Markes in der postembryonalen Entwicklung hin.

Daher kann man wohl sagen, dass das Volumen des Markes beim erwachsenen Tiere nicht weiter wächst oder nur sehr langsam sich vermehrt. Die individuelle Verschiedenheit der Organgrösse ist also hauptsächlich von der Menge der Rinde abhängig. 
In der vorliegenden Untersuchung habe ich das Mengenverhältnis des Markes und der Rinde beim ausgewachsenen Kaninchen eingehend studiert.

\section{Material und Methode.}

Nach Tsukamoto (27) beginnt die Pubertät des Kaninchens in 80-90 Tagen nach der Geburt, wobei das Körpergewicht durchschnittlich auf $1200 \mathrm{~g}$ sich beläuft. Ich habe so 27 normale erwachsene Kaninchen $(13 \hat{\delta}, 14$ ㅇ ), die etwa 2000 gr schwer waren, untersucht. Stilling (98) und Bager (17) sagen, dass die Nebemiere des Kaninchens je nach den Jahreszeiten eine Volumenveränderung zeigt; ich tötete daher die Tiere alle gleichzeitig (im Januar 1928). Im roraus fütterte ich die Tiere etwa 10 Tage mit ganz gleicher Nahrung unter denselben Bedingungen, dann narkotisierte ich sie mit Chloroform und exstirpierte die Nebennieren von der Umgebung möglichst frei präpariert.

Die Organe wurden sofort frei mit der Torsionswage und dann in physiologischer Kochsalzlösung ınit der chemischen Wage gewogen, um die Volumina der frischen Organe nach Stölzner (06) zu berechnen, wie folgt:

$$
x=\frac{(p-q) \times 100}{r} .
$$

$x=$ Volumen der frischen Nebenniere.

$p=$ Gewicht der frischen Nebenniere in Luft.

$q=$ Gewicht der frischen Nebenniere in physiologischer Kochsalzlösung.

$r=$ Gewicht der 100 ce physiologischen Kochsalzlösung.

$p-q=$ Gewichtverlust der frischen Nebemiere in physiologischer Kochsalzlösung, gleich dem Gewicht der durch die Nebenniere verdrängten Kochsalzlösung.

Für die volumetrische Untersuchung sind solche Fixierungsmittel am besten, welche den Organen die geringste Volumveränderung gestatten. Stölzner hat über die Beeinflussung der Fixierungsmittel auf die Organe eine eingehende Studie gemacht und die gesättigte Sublimatlösung mit 4\% Rohrzucker als ein solches Fixierungsmittel betrachtet, das den Organen die geringste Volumveränderung gestattet. Auch ich verfolgte wie Stölzner(06) die Volumveränderung der Nebennieren in 
Quantitative Studie des Nebennierenmarks und -rinde heim Kaninchen.

verschiedenen Fixierungsmitteln und absolutem Alkohol, dessen Ergebnis in Tabelle I zusammengestellt ist.

Table I.

\begin{tabular}{|c|c|c|c|c|c|c|}
\hline Nr. (Seite) & $\begin{array}{l}10 \% \text { Formol- } \\
\text { lösung }\end{array}$ & $\begin{array}{c}10 \% \text { Formol- } \\
\text { lösung mit } \\
4 \% \text { Rohr- } \\
\text { \%ucker }\end{array}$ & $\begin{array}{l}\text { Ortsche } \\
\text { Jösung }\end{array}$ & $\begin{array}{c}\text { M ïlle rsche } \\
\text { Jösung }\end{array}$ & $\begin{array}{l}\text { Gesättigte } \\
\text { Sublimat- } \\
\text { lösung mit } \\
4 \% \text { Rohr- } \\
\text { zucker }\end{array}$ & $\begin{array}{c}\text { Absoluter } \\
\text { Alkohol }\end{array}$ \\
\hline $12(\mathrm{r})$ & $+16 \%$ & 一 & - & - & - & $-22 \%$ \\
\hline $12(1)$ & $+16 \%$ & - & - & - & - & $-24 \%$ \\
\hline $13(r)$ & $+10 \%$ & - & - & - & - & $-23 \%$ \\
\hline $13(1)$ & $+10 \%$ & - & - & - & - & $-24 \%$ \\
\hline $14(\mathrm{r})$ & $+16 \%$ & - & 一 & - & - & $-26 \%$ \\
\hline $14(1)$ & $+19 \%$ & - & - & - & - & $-27 \%$ \\
\hline $15(r)$ & - & $+2.5 \%$ & - & - & - & $-26 \%$ \\
\hline $15(1)$ & - & $+4.6 \%$ & - & - & - & $-29 \%$ \\
\hline $19(r)$ & - & 一 & $-24 \%$ & $-21 \%$ & 一 & $-29 \%$ \\
\hline $19(1)$ & - & - & $-19 \%$ & $-17 \%$ & - & $-25 \%$ \\
\hline $16(\mathrm{r})$ & 一 & - & - & - & $-12 \%$ & $-33 \%$ \\
\hline $16(1)$ & - & - & 一 & - & $-13 \%$ & $-34 \%$ \\
\hline $8(r)$ & - & - & - & 一 & $-4.7 \%$ & \\
\hline
\end{tabular}

So zeigen die fixierten Organe die Volumveränderung in verschiedenen Graden. Im absoluten Alkohol ist aber die Schrumpfung fast konstant, durchschnittlich $27 \%$ Schrumpfung, zu finden. Die Organe $\mathrm{Nr}$. 15 zeigen die geringste Volumveränderung in Fixierungsflüssigkeit, aber im absoluten Alkohol dieselbe Schrumpfung wie die Organe bei anderen Fixierungen; daher glaubte ich, dass jedes Fixierungsmittel zum 'Zweck der volumetrischen Untersuchung gebraucht werden kann. So fixierte ich den grössten Teil des Materials in 10\%iger Formollösung. 
Die Organe wurden in Zelloidin eingebettet, senkrecht zu ihrer Längsachse $30 \mu$ dick geschnitten und die Schnitte mit Hämatoxylin und Eosin gefärbt.

Eine Anzahl ron Autoren wie Elliot (06), Herring (20), Jaffe und Marine (23), Castaldi (22) bestätigten die Vergrösserung der Nebenniere der Ratte und des Kaninchens bei der Schwangerschaft, doch Donaldson (24) fand bei der Ratte keine Zunahme der Grösse. Neuerdings hat Masui (25) im Gegensatz dazu vielmehr die Verminderung des Nebennierengewichts bei der Maus beobachtet. Wenn die Nebenniere volumetrisch durch Schwangerschaft beeinflusst wird so müssen auch das Mark und die Rinde eine Volumveränderung erleiden, dachte Elliot (06). Ich durchmusterte bei Exstirpation der Nebennieren vorsichtig den Uterus und konnte bei allen untersuchten Weibchen kein Schwangerschaftzeichen finden.

Zur Volumbestimmung wurde die Ausschneidungsmethode benutzt; aber anstatt der bisher im allgemeinen ausgeführten abgekürzten Methode wurden hier alle Schnitte der lückenlosen Serien bei 50 facher Vergrösserung auf gleich dickes Papier projiziert und abgezeichnet, wobei die ganze äussere Kontur des Schnittes, die der Rinde und die Grenze zwischen den beiden Substanzen des Organes, auch die Gefässe in den beiden und die Rindeninseln im Mark, möglichst genau umfahren wurden. Aus den Zeichnungen wurde jede Abteilung mit der Schere ausgeschnitten und auseinander gewogen, wobei die Gefässe zu dem Anhangsgebilde in der Umgebung des Organes und die Rindeninseln zur Rinde zugerechnet wurden. Aus der Summe des Papiergewichts des Markes, der Rinde und des Bindegewebes wurden die Volumina der beiden ersteren, mit dem des frischen Organs vergleichend, prozentuell ausgerechnet.

Meine Methode weicht von den bisher angefürten in folgenden Punkten ab. Ich habe zuerst das Volumen des frischen Organs gewogen, während bisher nur das Gewicht gewogen wurde. Ferner habe ich die Menge des umgebenden Bindegewebes, das in der Tatsache grösser als das Mark ist, berechnet, was auch bisher ganz ausser acht gelassen worden war. Endlich habe ich alle Schnitte der lückenlosen Serien gezeichnet und berechnet, während in den bisherigen Untersuchungen nur wenige Schnitte gezeichnet worden sind und von dessen Durchschnittswert die Menge des ganzen Organs ausgerechnet wurde.

Es steht ausser allem Zweifel, dass die direkt von geschrumpften Präparaten erhaltenen Werte viel geringer als die von den frischen Organen sind. Bei der quantitativen Forschung der Hypophyse fand 
Jackson (19) 50\% Schrumpfung an den vom in Zenkerschen Lösung fixierten und in Paraffin einbetteten Material angefertigten Präparaten. Auch nach der Mitteilung des Wistar Institutes beläuft die Schrumpfung sich auf 41\%. Akiyama (28) fand auch 32.5\% Schrumpfung bei derselben Fixierung und denselben Paraffinschnitten der Nebennieren von Ratte.

Tabelle II.

\begin{tabular}{|c|c|}
\hline$I r$ (Seite) & Schrumpfung \\
& $28 \%$ \\
\hline $12(\mathrm{r})$ & $26 \%$ \\
\hline $13(\mathrm{r})$ & $30 \%$ \\
\hline $13(\mathrm{l})$ & $29 \%$ \\
\hline $11(\mathrm{r})$ & $33 \%$ \\
\hline $11(\mathrm{l})$ & $48 \%$ \\
\hline $25(\mathrm{r})$ & $39 \%$ \\
\hline $25(\mathrm{l})$ & $22 \%$ \\
\hline $27(\mathrm{r})$ & $36 \%$ \\
\hline $27(\mathrm{I})$ & $38 \%$ \\
\hline
\end{tabular}

Ich konstatierte auch an meinen Materialien die Schrumpfung, die individuell ziemlich schwankt (Tabelle II). Diese individuelle Schwankung der Schrumpfung bei der Fixierung ist immer wohl im Auge zu behalten.

Die Mengen des Markes und der Rinde, die aus dem prozentuellen Verhältnis der Schnitte und des frischen Organgewichtes ausgerechnet sind, sind viel exakter als die nur direkt von geschrumpften Präparaten berechneten, demn das in der Umgebung des Organs liegende Bindegewebe hat relativ leichteres Gewicht und dadurch, wemn die beiden Bestandteile in Gewicht angegeben werden, kann das Bindegewebe leicht ausser acht gelassen werden. Doch das Mark und die Rinde haben jede für sich eine verschiedene Densität; die beiden werden also richtiger in Volumen als in Gewicht gezeichnet. Auch gibt dieselbe Methode grössere Werte als bei der meinigen, weil das Bindegewebe in der Umgebung des Organs frisch zusammen gewogen, aber nachher nicht angegeben ist.

Ferner sind bei den lisherigen Arbeiten die im Mark eingedrungenen Rindeninseln und die Gefässe des Markes und der Rinde wenig berücksichtigt, obgleich einige Autoren darauf hingewiesen hatten, doch beschränkte es sich nur auf die grossen Gefässe; ich habe jedoch darauf meine besondere Aufmerksamkeit gerichtet.

\section{Befund und Zusammenstellung.}

1) Gewicht und spezifisches Gewicht der Nebenniere. 
Tabelle III.

\begin{tabular}{|c|c|c|c|c|c|c|c|}
\hline Nr. & Seite & $\begin{array}{c}\text { Ge- } \\
\text { schlecht }\end{array}$ & $\begin{array}{l}\text { Körper- } \\
\text { gewicht } \\
\text { (g) }\end{array}$ & $\begin{array}{l}\text { Nehen- } \\
\text { nieren- } \\
\text { gewicht } \\
(\mathrm{g})\end{array}$ & $\begin{array}{l}\text { Seben- } \\
\text { nieren- } \\
\text { volumen } \\
\text { (cc) }\end{array}$ & $\begin{array}{l}\text { Spezifisches } \\
\text { Gewicht der } \\
\text { Nebenniere }\end{array}$ & $\begin{array}{l}\text { Neben- } \\
\text { nieren- } \\
\text { gewicht } \\
\text { pro mille } \\
\text { Körper- } \\
\text { gewicht } \\
\text { (g) }\end{array}$ \\
\hline 1 & $\begin{array}{l}r \\
1\end{array}$ & $\hat{o}$ & 2170 & $\begin{array}{l}0.155 \\
0.155\end{array}$ & $\begin{array}{l}0.151 \\
0.150\end{array}$ & $\begin{array}{l}1.0283 \\
1.0851\end{array}$ & $0.1+80$ \\
\hline$\ddot{2}$ & $\begin{array}{l}x \\
1\end{array}$ & $q$ & 2180 & $\begin{array}{l}0.219 \\
0.237\end{array}$ & $\begin{array}{l}0.210 \\
0.228\end{array}$ & $\begin{array}{l}1.0417 \\
1.0391\end{array}$ & 0.2091 \\
\hline 3 & $\begin{array}{l}r \\
1\end{array}$ & $\hat{\delta}$ & 2185 & $\begin{array}{l}0.165 \\
0.176\end{array}$ & $\begin{array}{l}0.161 \\
0.171\end{array}$ & $\begin{array}{l}1.0271 \\
1.0818\end{array}$ & 0.1557 \\
\hline 4 & $\begin{array}{l}r \\
l\end{array}$ & $\hat{o}$ & 2240 & $\begin{array}{l}0.177 \\
0.416\end{array}$ & $\begin{array}{l}0.171 \\
0.407\end{array}$ & $\begin{array}{l}1.0318 \\
1.0232\end{array}$ & 0.2690 \\
\hline 5 & $\begin{array}{l}r \\
1\end{array}$ & q & 2870 & $\begin{array}{l}0.283 \\
0.290\end{array}$ & $\begin{array}{l}0.275 \\
0.283\end{array}$ & $\begin{array}{l}1.0298 \\
1.0297\end{array}$ & 0.2421 \\
\hline 6 & $\begin{array}{l}r \\
1\end{array}$ & $\hat{o}$ & 2835 & $\begin{array}{l}0.182 \\
0.191\end{array}$ & $\begin{array}{l}0.175 \\
0.183\end{array}$ & $\begin{array}{l}1.0427 \\
1.0408\end{array}$ & 0.1669 \\
\hline 7 & $\begin{array}{l}r \\
1\end{array}$ & o & 2325 & $\begin{array}{l}0.186 \\
0.184\end{array}$ & $\begin{array}{l}0.180 \\
0.177\end{array}$ & $\begin{array}{l}1.0305 \\
1.0483\end{array}$ & 0.1592 \\
\hline 8 & $\begin{array}{l}r \\
1\end{array}$ & 우 & 2270 & $\begin{array}{l}0.395 \\
0.312\end{array}$ & $\begin{array}{l}0.287 \\
0.303\end{array}$ & $\begin{array}{l}1.0293 \\
1.0282\end{array}$ & 0.2673 \\
\hline 9 & $\begin{array}{l}r \\
1\end{array}$ & 우 & 2907 & $\begin{array}{l}0.265 \\
0.244\end{array}$ & $\begin{array}{l}0.259 \\
0.2369\end{array}$ & $\begin{array}{l}1.0199 \\
1.0295\end{array}$ & 0.2205 \\
\hline 10 & $\begin{array}{l}r \\
1\end{array}$ & $\hat{o}$ & 2930 & $\begin{array}{l}0.165 \\
0.182\end{array}$ & $\begin{array}{l}0.157 \\
0.173\end{array}$ & $\begin{array}{l}1.0531 \\
1.0488\end{array}$ & 0.1556 \\
\hline
\end{tabular}


Tabelle III. (Fortsetzung).

\begin{tabular}{|c|c|c|c|c|c|c|c|}
\hline $\mathrm{Nr}$ & Seite & $\begin{array}{c}\text { Ge } \\
\text { schlecht }\end{array}$ & $\begin{array}{l}\text { Körper- } \\
\text { gewicht } \\
\text { (g) }\end{array}$ & $\begin{array}{l}\text { Neben- } \\
\text { nieren- } \\
\text { gewicht } \\
\text { (g) }\end{array}$ & $\begin{array}{l}\text { Neben- } \\
\text { nieren- } \\
\text { volumen } \\
\text { (cc) }\end{array}$ & $\begin{array}{l}\text { Spezifisches } \\
\text { Gewicht der } \\
\text { Nebenniere }\end{array}$ & $\begin{array}{l}\text { Neben- } \\
\text { nieren- } \\
\text { gewicht } \\
\text { pro mille } \\
\text { Körper- } \\
\text { gewicht } \\
\text { (g) }\end{array}$ \\
\hline 11 & $\begin{array}{l}r \\
1\end{array}$ & $\hat{\delta}$ & 2489 & $\begin{array}{l}0.185 \\
0.227\end{array}$ & $\begin{array}{l}0.178 \\
0.220\end{array}$ & $\begin{array}{l}1.0364 \\
1.0311\end{array}$ & 0.1655 \\
\hline ]: & $\begin{array}{l}\mathbf{r} \\
1\end{array}$ & q & 1907 & $\begin{array}{l}0.150 \\
0.174\end{array}$ & $\begin{array}{l}0.143 \\
0.167\end{array}$ & $\begin{array}{l}1.0431 \\
1.0382\end{array}$ & 0.1699 \\
\hline 18 & $\begin{array}{l}r \\
1\end{array}$ & q & 2034 & $\begin{array}{l}0.308 \\
0.341\end{array}$ & $\begin{array}{l}0.300 \\
0.328\end{array}$ & $\begin{array}{l}1.0251 \\
1.0389\end{array}$ & 0.3190 \\
\hline 14 & $\begin{array}{l}r \\
1\end{array}$ & $\hat{\delta}$ & 2452 & $\begin{array}{l}0.189 \\
0.188\end{array}$ & $\begin{array}{l}0.178 \\
0.177\end{array}$ & $\begin{array}{l}1.0589 \\
1.0591\end{array}$ & 0.1538 \\
\hline 1.5 & $\begin{array}{l}r \\
1\end{array}$ & 우 & 2374 & $\begin{array}{l}0.3625 \\
0.331\end{array}$ & $\begin{array}{l}0.351 \\
0.321\end{array}$ & $\begin{array}{l}1.0327 \\
1.0302\end{array}$ & 0.2920 \\
\hline 16 & $\begin{array}{l}r \\
1\end{array}$ & $\hat{\delta}$ & 2296 & $\begin{array}{l}0.3385 \\
0.333\end{array}$ & $\begin{array}{l}0.3265 \\
0.320\end{array}$ & $\begin{array}{l}1.0369 \\
1.0387\end{array}$ & 0.2924 \\
\hline 17 & $\begin{array}{l}r \\
1\end{array}$ & 우 & 2217 & $\begin{array}{l}0,102 \\
0.120\end{array}$ & $\begin{array}{l}0.099 \\
0.115\end{array}$ & $\begin{array}{l}1.0286 \\
1.0431\end{array}$ & 0.1001 \\
\hline 18 & $\begin{array}{l}r \\
1\end{array}$ & ㅇ & 2210 & $\begin{array}{l}0.141 \\
0.180\end{array}$ & $\begin{array}{l}0.134 \\
0.172\end{array}$ & $\begin{array}{l}1.0455 \\
1.0431\end{array}$ & 0.1452 \\
\hline 19 & $\begin{array}{l}r \\
1\end{array}$ & $\hat{\jmath}$ & 1770 & $\begin{array}{l}0.382 \\
0.398\end{array}$ & $\begin{array}{l}0.366 \\
0.383\end{array}$ & $\begin{array}{l}1.0411 \\
1.0374\end{array}$ & 0.4406 \\
\hline 20 & $\begin{array}{l}r \\
1\end{array}$ & $\hat{o}$ & $\begin{array}{c}2105 \\
:\end{array}$ & $\begin{array}{l}0.253 \\
0.244\end{array}$ & $\begin{array}{l}0.237 \\
0.230\end{array}$ & $\begin{array}{l}1.0674 \\
1.0590\end{array}$ & 0.2360 \\
\hline
\end{tabular}


Tabelle III. (Fortsetzung).

\begin{tabular}{|c|c|c|c|c|c|c|c|}
\hline Nr. & Seite & $\begin{array}{c}\mathrm{Ge}- \\
\text { schlecht }\end{array}$ & $\begin{array}{l}\text { Körper- } \\
\text { gewicht } \\
\text { (g) }\end{array}$ & $\begin{array}{l}\text { Jeben- } \\
\text { nieren- } \\
\text { gewicht } \\
\text { (g) }\end{array}$ & $\begin{array}{l}\text { Neben- } \\
\text { nieren } \\
\text { volumen } \\
\text { (cc) }\end{array}$ & $\begin{array}{l}\text { Npezifisches } \\
\text { (rewicht der } \\
\text { Nebenniere }\end{array}$ & $\begin{array}{l}\text { Neben- } \\
\text { nieren- } \\
\text { gewicht } \\
\text { pro mille } \\
\text { Körper- } \\
\text { gewicht } \\
\text { (g) }\end{array}$ \\
\hline 21 & $\begin{array}{l}r \\
1\end{array}$ & $\delta$ & 2408 & $\begin{array}{l}0.088 \\
0.101\end{array}$ & $\begin{array}{l}0.084 \\
0.096\end{array}$ & $\begin{array}{l}1.0439 \\
1.0500\end{array}$ & 0.0785 \\
\hline 22 & $\begin{array}{l}r \\
1\end{array}$ & $\hat{\delta}$ & 2110 & $\begin{array}{l}0.105 \\
0.129\end{array}$ & $\begin{array}{l}0.101 \\
0.124\end{array}$ & $\begin{array}{l}1.0379 \\
1.0325\end{array}$ & 0.1109 \\
\hline 23 & $\begin{array}{l}r \\
1\end{array}$ & ㅇ & 1775 & $\begin{array}{l}0.094 \\
0.108\end{array}$ & $\begin{array}{l}0.090 \\
0.105\end{array}$ & $\begin{array}{l}1.0416 \\
1.0274\end{array}$ & 0.11 is \\
\hline 24 & $\begin{array}{l}r \\
1\end{array}$ & ㅇ․ & 2475 & $\begin{array}{l}0.305 \\
0.316\end{array}$ & 0.302 & 1.0448 & 0.2508 \\
\hline 25 & $\begin{array}{l}r \\
1\end{array}$ & 우 & 2530 & $\begin{array}{l}0.233 \\
0.246\end{array}$ & $\begin{array}{l}0.228 \\
0.240\end{array}$ & $\begin{array}{l}1.0216 \\
1.0379\end{array}$ & 0.1893 \\
\hline 26 & $\begin{array}{l}\mathbf{r} \\
1\end{array}$ & ㅇ & 2235 & $\begin{array}{l}0.191 \\
0.235\end{array}$ & $\begin{array}{l}0.189 \\
0.232\end{array}$ & $\begin{array}{l}1.0089 \\
1.0128\end{array}$ & 0.1906 \\
\hline 27 & $\begin{array}{l}r \\
1\end{array}$ & $\hat{o}$ & 1970 & $\begin{array}{l}0.097 \\
0.181\end{array}$ & $\begin{array}{l}0.093 \\
0.195\end{array}$ & $\begin{array}{l}1.0406 \\
1.0485\end{array}$ & 0.1157 \\
\hline 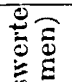 & & $\hat{\delta}+\hat{o}$ & 2217 & 0.436 & 0.4071 & 1.0371 & 0.1982 \\
\hline 焉 & & $\hat{\delta}$ & 2204 & 0.411 & 0.3944 & & 0.1910 \\
\hline 苾 & & ㅇ & 2.29 & 0.461 & 0.4190 & & 0.2049 \\
\hline
\end{tabular}

a) Nebennierengewicht.

Wie in Tabelle III gezeigt, sind das Gewicht und Volumen der Nebenniere nicht immer proportional zum Körpergewicht und schwanken individuell. Im folgenden wird ein Paar der beiderseitigen Nebennieren 
als Einheit zusammen gewogen gezählt. Ich bekam durchschnittlich das absolute Nebemnierengewicht von $0.436 \mathrm{~g}(0.411 \mathrm{~g} \hat{\delta}, 0.461 \mathrm{~g}$ \% $)$ und das relative von $0.1982 \mathrm{~g}(0.1910 \mathrm{~g} \hat{\delta}, 0.2049 \mathrm{~g}$ 우 $)$ pro Kilogramm Körpergewicht; also sind diese zweierlei Gewichte bein Weibchen durchschnittlich grösser als beim Männchen. In derselben Tabelle sieht man, dass die Gewichte bei den drei nach dem gleichen oder sehr nah stehenden Körpergewicht eingeteilten Gruppen (Nr. 2, Nr. 3), (Nr. 6. Nr. 26), (Nr. 11, Nr. 24) auch beim Weibchen viel grösser sind. Bager (17) fand auch beim Kaninchen dasselbe, 4 Monate und 18-25 Monate alte Tiere ausgenommen, und sagt, dass, ob das ungekehrte Verhältnis bei 18-25 Monate alten Tieren zufällig ist oder nicht, unklar ist; aber er glaubt im ganzen an die überwiegenden Gewichte beim Weibchen nach der Pubertät.

Aber die Resultate von vielen Autoren stimmen mit denen von Bager (17) und mir nicht überein, wie Tabelle IV zeigt. Denn nach Stewart und Rogoff (21), Takahashi (21) und Kojima (29) herrscht eine Überwiegung des absoluten und relativen Gewichtes beim Männchen und nach Boycott und Kellaway (24) und nach Imamura (24) ist das relative Organgewicht pro Kilogramm Körpergewicht beim Weibchen grösser als beim Männchen, wenngleich das umgekehrte Verhältnis im absoluten Durchschnittsgewicht der Organe wahrgenommen wird. Ob diese abweichenden Resultate auf der Individualität beruhen oder durch die Auswahlsweise der Tiere hervorgerufen werden, ist schwer zu entscheiden. Diese Frage zu lösen bedarf es noch weiterer Untersuchungen. Bei der Ratte erkennt Donaldson (19) auch den Geschlechtsunterschied an und sagt, dass das Organgewicht beim Weibchen immer das des Männchens mit gleichem Körpergewicht überwiegt, was im Alter immer mehr hervortritt. Dagegen ist nach Jackson (19) und Akiyama (28) kein Geschlechtsunterschied zu konstatieren. Elliot (06) sagt, dass beim Weibchen die Organgrösse wegen der beschleunigenden Wirkung der Schwangerschaft auf kurze Zeit nach Pubertät die des Männchens überwiegt, wobei hauptsächlich die Rinde und vielleicht auch das Mark, wenn auch nur sehr gering, sich vergrössern; aber später wird kein Unterschied festgestellt. An den Mäusen fand Hett (28), dass beim Weibchen das Gewicht der beiden Nebennieren zusammen grösser als beim Männchen ist, über $13 \mathrm{~g}$ Körpergewicht betragendes Weibchen ausgeschlossen, weil das Organgewicht sich durch die Gravidität verändern kamn. Er hält für das grössere Gewicht der weiblichen Nebenniere hauptsächlich die Zunahme der Rindensubstanz verantwortlich und sagt, dass die grössere Rinden- 
Tabelle IV.

\begin{tabular}{|c|c|c|c|c|c|}
\hline Autor & Zahl & Geschlecht & $\begin{array}{c}\text { Körper- } \\
\text { gewicht } \\
\text { (Kilogramm) }\end{array}$ & $\begin{array}{c}\text { Beiderseit. } \\
\text { Nebennieren- } \\
\text { gewicht } \\
\text { (Gramm) } \\
\text { (Durchschnitt) }\end{array}$ & $\begin{array}{l}\text { Beiders. Neben- } \\
\text { nierengewicht } \\
\text { pro Kilogramm } \\
\text { Körpergewicht } \\
\text { (Gramm) } \\
\text { (Durchschnitt) }\end{array}$ \\
\hline Herring (16) & 3 & & $1.8 \quad-2.4$ & 0.399 & 0.200 \\
\hline Kurigama (18) & 6 & $1 \delta, \begin{array}{l}5 \% \\
5 \hat{\circ}\end{array}$ & $\begin{array}{l}1.46-2.4 \\
1.46-2.4\end{array}$ & $\begin{array}{l}0.380 \\
0.397\end{array}$ & $\begin{array}{l}0.397 \\
0.204\end{array}$ \\
\hline Midorikawa (19) & 14 & & $1.70-2.47$ & $0.5 \pm 5$ & 0.236 \\
\hline $\begin{array}{l}\text { Stewart } \\
\text { goff }(21)\end{array}$ Ro- & 15 & $\begin{array}{r}11 \delta, 4 \text { 우 } \\
11 \text { 우 } \\
4 \text { 우 }\end{array}$ & $\begin{array}{l}1.48-3.01 \\
1.48-3.01 \\
2.06-2.52\end{array}$ & $\begin{array}{l}0.428 \\
0.448 \\
0.374\end{array}$ & $\begin{array}{l}0.176 \\
0.182 \\
0.160\end{array}$ \\
\hline Specht $(21)$ & 8 & $4 \hat{0}, 4$ ㅇ & $1.50-3.60$ & $0.43 \pm 5$ & 0.1604 \\
\hline Takahashi (21) & 10 & 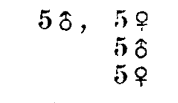 & $\begin{array}{l}1.52-2.20 \\
1.58-2.15 \\
1.52-2.20\end{array}$ & $\begin{array}{l}0.323 \\
0.350 \\
0.292\end{array}$ & $\begin{array}{l}0.179 \\
0.197 \\
0.161\end{array}$ \\
\hline $\begin{array}{l}\text { Squier u. (irab- } \\
\text { field (22) }\end{array}$ & 14 & & & 0.294 & 0.169 \\
\hline Morita (23) & 7 & & $1.5-2.9$ & 0.869 & 0.191 \\
\hline $\begin{array}{l}\text { Boycott w. Kel. } \\
\text { laway (24) }\end{array}$ & 20 & 120, $\begin{array}{r}89 \\
120 \\
89\end{array}$ & $\begin{array}{l}1.61-3.64 \\
1.61-3.64 \\
2.30-3.34\end{array}$ & $\begin{array}{l}0.650 \\
0.648 \\
0.660\end{array}$ & $\begin{array}{l}0.250 \\
0.247 \\
0.239\end{array}$ \\
\hline Kisch (24) & 28 & 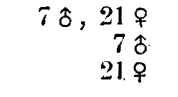 & & & $\begin{array}{l}0.1779 \\
0.1450 \\
0.1841\end{array}$ \\
\hline Imamua & 57 & $\begin{array}{r}38 \text { ô, } 19 \text { 우 } \\
38 \text { 연 } \\
19 \text { 우 }\end{array}$ & $\begin{array}{l}1.60-2.50 \\
1.60-2.46 \\
1.85-2.50\end{array}$ & $\begin{array}{l}0.41 \\
0.407 \\
0.418\end{array}$ & $\begin{array}{l}0.191 \\
0.193 \\
0.187\end{array}$ \\
\hline Fujii $(24)$ & 150 & nur $\hat{o}$ & $1.3-2.6$ & 0.402 & \\
\hline $\begin{array}{l}\text { Brown, Pearceu. } \\
\text { ran lllen }(0(6)\end{array}$ & $64 t$ & nur $\hat{o}$ & $1.4-3.5$ & 0.3833 & 0.2082 \\
\hline Tsukamoto & & $\begin{array}{l}\hat{0}+q \\
\delta+q \\
\delta+q\end{array}$ & $\begin{array}{l}1.208 \\
1.610 \\
1.175\end{array}$ & $\begin{array}{l}0.40 \\
0.40 \\
0.40\end{array}$ & $\begin{array}{l}0.1208 \\
0.1610 \\
0.1750\end{array}$ \\
\hline Kojima $(29)$ & 200 & $\begin{array}{r}100 \text { ㅇ, } 100 \text { 우 } \\
100 \text { 오 } \\
100 \text { 우 }\end{array}$ & $\begin{array}{l}1.07-3.99 \\
1.07-3.90 \\
1.15-3.99\end{array}$ & $\begin{array}{l}0.276 \\
0.297 \\
0.255\end{array}$ & $\begin{array}{l}0.159 \\
0.167 \\
0.151\end{array}$ \\
\hline Onozawa & 27 & $13 \hat{\delta}, \begin{array}{l}14 \text { 우 } \\
13 \hat{\delta} \\
14 ㅇ\end{array}$ & $\begin{array}{l}1.77-2.53 \\
1.77-2.453 \\
1.907-2.53\end{array}$ & $\begin{array}{l}0.436 \\
0.411 \\
0.461\end{array}$ & $\begin{array}{l}0.1982 \\
0.1910 \\
0.2049\end{array}$ \\
\hline
\end{tabular}


menge dadurch bedingt werde, dass nicht allein die Nebenniere des Weibchens im ganzen grösser ist, sondern auch die prozentuale Zusammensetzung des Organes bei beiden Geschlechtern verschieden ist. Ferner äussert er, „Ob sich die grössere Rindenmenge des Weibchens während der weiteren postembryonalen Entwicklung ausgleicht, kamn bis jetzt noch nicht sicher ángegeben werden. Ich glaube jedoch dies aus einigen hier nicht ausgeführten Zahlen älterer Weibchen verneinen zu müssen. Wenn wir uns nun fragen, welche Bedeutung hat die grössere Rindenmenge des weiblichen Tieres, so lässt sich dies vorerst nicht eindeutig beantworten. Man ist zunächst geneigt, die grössere Rindenmenge des Weibchens mit der Gravidität in Zusammenhang zu bringen. So soll sich in der Schwangerschaft hauptsächlich die Rinde vergrössern und die allgemeine Zunahme des Organes bedingen."

Beim Meerschweinchen beobachtet Castaldi (22) auch die Überwiegung des absoluten Organgewichts des Weibchens und betreffs desselben pro Kilogramm Körpergewicht das umgekehrte Verhältnis.

Die absoluten Nebennierengewichte zeigen erhebliche Schwankung und dies kann daraus leicht verstanden werden, dass die absoluten Durchschnittswerte der Nebemieren nach Autoren voneinander abweichen, wie in Tabelle IV gezeigt. Aber als Durchschnittsgewicht der Nebennieren pro Kilogramm Körpergewicht geben die meisten Autoren etwa $0.2 \mathrm{~g}$ an ; besonders gleicht das Resultat Imamuras (24) dem meinen sehr, bezüglich des Körpergewichtes der untersuchten Tiere, des Durchschnittsgewichtes der Nebenniere u.a.m. Daher ist es vielleicht richtig, las Organgewicht pro Kilogramm Körpergewicht beim erwachsenen Kaninchen durchschnittlich für etwa $0.2 \mathrm{~g}$ zu halten.

Betreffs des beiderseitigen Nebemnierengewichtes haben viele Autoren die Erfahrung gemacht, dass die linke viel schwerer als die rechte ist. Bei meiner Untersuchung ist die gleiche Tatsache bei 20 Fällen unter 27 Tieren beobachtet worden, während bei 6 Fällen die rechte schwerer und bei einem Fall die beiderseitigen gleich schwer waren (Tabelle V). J)a Nr. 4 einen bedeutenden Unterschied nach den Seiten hin aufweist, so wurde dieser Fall bei Berechnung des Überwiegunsprozentsatzes ausgeschlossen. Der letztere ist einer individuellen Schwankung unterworfen, aber ist im ganzen links grösser als rechts. Die Frequenz der Überwiegung der beiderseitigen Nebennieren ist nach 8 Forschern durchschnittlich links $84.2 \%$ und rechts 15.8\% (Tabelle V, 393 Tiere, $\mathrm{L} \geqslant \mathrm{R}=331, \mathrm{R}>\mathrm{L}=62$ ), Stilling (98), Kisch (24) und Fujii (24) (24) ausgenommen. So kann man die Überwiegung der linken Nebennierengrösse als die Regel ansehen. 
Tabelle V.

\begin{tabular}{|c|c|c|c|c|c|c|c|}
\hline Autor & Zahl & $\begin{array}{c}\text { Rechtes } \\
\text { Neben- } \\
\text { nieren- } \\
\text { gewicht } \\
\text { (g) } \\
\text { (Durch- } \\
\text { schnitt) }\end{array}$ & $\begin{array}{l}\text { Linkes } \\
\text { Neben- } \\
\text { nieren- } \\
\text { gewicht } \\
\text { (g) } \\
\text { (Durch- } \\
\text { schnitt) }\end{array}$ & $\begin{array}{c}\mathrm{L}>\mathrm{R} \\
\% \\
(\mathrm{Zahl})\end{array}$ & $\begin{array}{c}\mathrm{R}>\mathrm{L} \\
\% \\
(\mathrm{Zahl})\end{array}$ & $\begin{array}{c}\text { Frequenz } \\
\text { von } \mathbf{L}=\mathbf{R} \\
\% \\
\text { (Zahl) }\end{array}$ & $\begin{array}{c}\text { Frequenz } \\
\text { von } \mathrm{R}>\mathrm{I} \\
\% \\
(\mathrm{Zahl})\end{array}$ \\
\hline Stilling & & & & $\mathrm{I}>\mathrm{R}$ & & & \\
\hline Kuriyama & 6 & 0.174 & 0.207 & $18.4(6)$ & & & \\
\hline Midorikawa & 14 & 0.257 & 0.268 & $8.2(11)$ & $9.9(3)$ & & \\
\hline $\begin{array}{c}\text { Stewart } \\
\text { Rogoff }\end{array}$ & & 0.206 & 0.222 & $11.0(13)$ & $6.5(2)$ & 86.7 & 13.3 \\
\hline Takahashi & 10 & 0.156 & 0.167 & $15.2(7)$ & $4.6(3)$ & 70.0 & 30.0 \\
\hline $\begin{array}{l}\text { Boycott u. } \\
\text { Kellaway }\end{array}$ & 20 & 0.306 & 0.346 & $16.8(17)$ & $8.3(3)$ & 85.0 & 15.0 \\
\hline $\mathrm{Kisch}$ & 28 & & & Max. 38.1 & & \multicolumn{2}{|c|}{$\begin{array}{l}\mathrm{L}>\mathrm{R} \text { in } \frac{2}{3} \\
\mathrm{I}=\mathrm{R} \text { in } \frac{1}{3}\end{array}$} \\
\hline Kojima & 200 & 0.130 & 0.143 & $11.8(16:)$ & $5.4(38)$ & 81.0 & 19.0 \\
\hline$F u j i i$ & 150 & 0.193 & 0.209 & & & 80.7 & 19.3 \\
\hline Bager & 101 & & & & & $\mid \begin{array}{c}94.1(94) \\
\text { in } 3 \text { davon } \\
\mathrm{L}=\mathrm{R}\end{array}$ & $5.9(7)$ \\
\hline Onozawa & 27 & 0.208 & 0.229 & $\begin{array}{l}14.7(19) \\
\operatorname{Max} .2 .4 \\
\text { Min. } 36.0\end{array}$ & $\begin{array}{c}4.0(6) \\
\text { Max. } 9.3 \\
\operatorname{Min.} 0.5\end{array}$ & $77.8(21)$ & $22.2(6)$ \\
\hline
\end{tabular}

Die Gewichtsdifferenz zwischen den beiderseitigen Nebennieren bei beiden Geschlechtern ist beim Weibchen (durchschnittlich $0.0208 \mathrm{~g}$ ) nur etwas grösser als beim Männchen (durchschnittlich $0.0151 \mathrm{~g}, \mathrm{Nr} .4$ wegen der Abnormität ausgenommen).

b) das spezifische Gewicht der Nebenniere.

Dies ist mehr oder weniger der individuellen Schwankung unterworfen, was nach meiner Meinung durch die Menge des in der Umgebung des Organs befindlichen Bindegewebes, des noch darin enthaltenen 
Blutes, das bei Exstirpation in verschiedenen Massen verloren geht, u.a. bedingt wird. Als Durchschnittswert des spezifischen Gewichtes kounte ich 1.0371 bekommen.

2) Mark und Rinde.

a) Mark.

Das Volumen des Markes ist nicht immer proportional zur Grösse der Nebenniere selbst und des Körpergewichtes. Das zeigt nicht so beträchtlichere individuelle Schwankung als das des ganzen Organs und hat einen ziemlich konstanten Wert. Das Mark ist bekanntlich sehr klein im Vergleich mit der Rinde und nimmt durchschnittlich nur 3.16\% Volumen des Organs ein, die beiderseitigen zusammen berechnet. Daher bin ich der Meinung, dass die Grösse der Nebenniere beim erwachsenen Kaninchen hauptsächlich von der Grösse der Rinde abbängig ist. Je grösser die Nebenniere ist, desto relativ kleineres Mark hat sie, wie der Nebenniere-Mark-Index es klar zeigt. Zum Beispiel ist die Nebenniere Nr. 15 etwa 3.5 mal grösser als die Nr. 21, wenngleich das Mark nur 1.6 mal grösser ist.

Das absolute Volumen des Marks ist durchschnittlich $0.0094 \mathrm{cc}$ (0.0087 ce beim Männchen, 0.0098 cc beim Weibchen), und der Geschlechtsunterschied ist nicht beträchtlich, wie wir auch bei Bager (17) sehen. Elliot (06) gibt als Durchschnittswert des Markes beim erwachsenen Kaninchen $0.01 \mathrm{~g}$ (die Zahl der untersuchten Tiere ist aber gering) und Bager (17) beim etwa 4 Monate alten Kaninchen $0.0098 \mathrm{~g}$, beim 12 Monate alten $0.0102-0.0119 \mathrm{~g}$, beim 21 Monate alten $0.0141 \mathrm{~g}$ und beim 42 Monate alten $0.0214 \mathrm{~g}$. Die Werte von diesen beiden Autoren sind dem meinen beinahe gleich, ausgenommen Bagers 21. Monate altes Tier. Die etwa grösseren Werte von ihnen wurden, denke ich, dadurch hervorgerufen, dass sie jeden 7 ten oder 10ten Schnitt der Serien abzeichneten, das umgebende Bindegewebe des Organes und die Gefässe sowie die Rindeninseln im Mark ausser acht liesen; ausserdem berechneten sie die Menge der beiden Substanzen in Gramm aus dem mittels Planimeterverfahren bekommenen Volumen und dem Organgewicht im frischen Zustand prozentuell. Die langsame, dauernde Vergrösserung des Markes nach der Pubertät kann man daraus vermuten, dass Bager (17) beim 3-6 Jahre alten Kaninchen das Marksgewicht von $0.0117-0.0379 \mathrm{~g}$ angibt.

Vergleicht man das Volumen des rechten Nebennierenmarkes mit dem des linken, so ergibt sich eine bemerkenswerte Tatsache, dass nämlich das Mark im allgemeinen in der Seite grösser ist, welche die grössere Nebenniere hat. In 10 Fällen ist solche Tatsache zu bemerken 


\begin{tabular}{|c|c|c|c|c|c|c|c|c|c|c|c|c|c|c|c|c|c|c|}
\hline \multicolumn{2}{|c|}{ Rindenmarkindex } & 茎 & 点 & $\stackrel{5}{S}$ & $\stackrel{5}{0}$ & 高 & 苋 & 竞 & 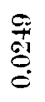 & 萨 & 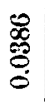 & 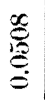 & 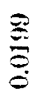 & 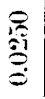 & 害 & 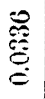 & 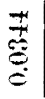 & 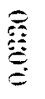 \\
\hline \multirow{5}{*}{ 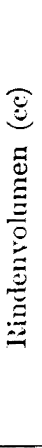 } & $\begin{array}{l}\% \text { d. Korper- } \\
\text { gewichts }\end{array}$ & $\frac{\pi}{2+\frac{1}{6}}$ & $\frac{\vec{\sigma}}{\frac{\pi}{0}}$ & 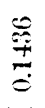 & 㫄 & 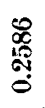 & 冚 & $\frac{7}{5}$ & $\begin{array}{l}\text { Lొ } \\
\text { ọ } \\
0 \\
0\end{array}$ & $\stackrel{\infty}{\mathscr{0}}$ & 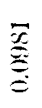 & 蓉 & $\frac{\vec{a}}{\frac{\pi}{c}}$ & స్ & $\stackrel{\infty}{8}$ & $\frac{1}{0}$ & $\frac{2}{20}$ & 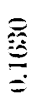 \\
\hline & $\begin{array}{c}\% \text { d. Neben- } \\
\text { nieren- } \\
\text { volumen }\end{array}$ & 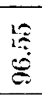 & 量 & $\begin{array}{l}\vec{s} \\
\stackrel{5}{5}\end{array}$ & 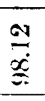 & 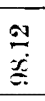 & 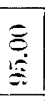 & 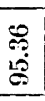 & $\begin{array}{l}5 \\
\stackrel{5}{5} \\
\end{array}$ & 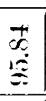 & 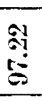 & 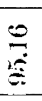 & $\begin{array}{l}\hat{S} \\
\stackrel{5}{E} \\
\end{array}$ & 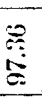 & 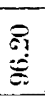 & 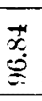 & 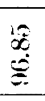 & 芯 \\
\hline & Beide & 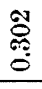 & în & $\stackrel{+}{6 !}$ & 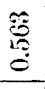 & $\stackrel{\pi}{0}$ & $\stackrel{5}{5}$ & : & 荘 & $\stackrel{\overrightarrow{0}}{0}$ & $\stackrel{5}{\stackrel{s}{: !}}$ & 萢 & $\begin{array}{l}\ddot{0} \\
0 \\
0\end{array}$ & 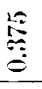 & 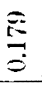 & 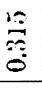 & 禺 & 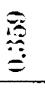 \\
\hline & Links & 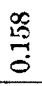 & 紊 & $\stackrel{\dddot{m}}{\stackrel{9}{\rightleftarrows}}$ & 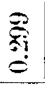 & 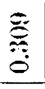 & 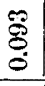 & $\frac{1}{0}$ & $\frac{x}{8 !}$ & 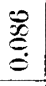 & 望 & 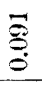 & $\stackrel{0}{\varrho !}$ & 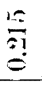 & $\begin{array}{l}\stackrel{1}{0} \\
0 \\
\end{array}$ & $\frac{10}{0+0}$ & $\begin{array}{l}\overline{\bar{s}} \\
=\end{array}$ & 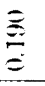 \\
\hline & Rechts & $\frac{j}{6}$ & 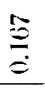 & 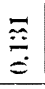 & 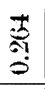 & 鹪 & $\stackrel{\pi}{c}$ & $\frac{\infty}{0}$ & 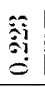 & $\begin{array}{l}19 \\
0 \\
0 \\
\end{array}$ & $\begin{array}{l}\hat{0} \\
\dot{0} \\
\dot{0}\end{array}$ & $\stackrel{x}{\mathscr{x}}$ & $\stackrel{0}{0}$ & $\begin{array}{l}8 \\
0 \\
\end{array}$ & 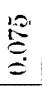 & 揨 & $\stackrel{\overline{\hat{i}}}{\bar{E}}$ & $\begin{array}{l}\tilde{\ddot{E}} \\
\stackrel{E}{E}\end{array}$ \\
\hline \multirow{5}{*}{ 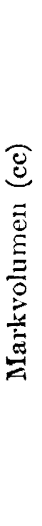 } & $\begin{array}{c}\% \text { d. Körper- } \\
\text { gewicht }\end{array}$ & 茰 & 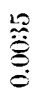 & 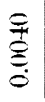 & 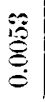 & 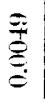 & $\begin{array}{l}\text { 管 } \\
\stackrel{8}{0}\end{array}$ & 芯 & 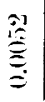 & $\begin{array}{c}\tilde{\hat{\tilde{i}}} \\
\stackrel{\delta}{\dot{\delta}}\end{array}$ & 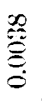 & 舫 & 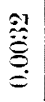 & 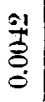 & 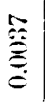 & 管 & 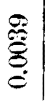 & 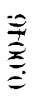 \\
\hline & $\begin{array}{c}\% \text { d. Neben- } \\
\text { nieren- } \\
\text { volumen }\end{array}$ & 19 & 柋 & $\stackrel{8}{:}$ & $\stackrel{\infty}{\propto}$ & $\begin{array}{l}\infty \\
\stackrel{\infty}{\infty} \\
\stackrel{\infty}{n}\end{array}$ & $\Xi_{i}$ & $\stackrel{+}{+}$ & ơ & $\stackrel{\circ}{\dddot{\sigma}}$ & 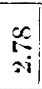 & $\vec{x}$ & 参 & $\underset{s}{+}$ & $\begin{array}{l}0 \\
\therefore\end{array}$ & $\stackrel{0}{\infty}$ & $\frac{19}{5}$ & $\stackrel{\Leftrightarrow}{\therefore}$ \\
\hline & Beide & $\stackrel{\infty}{\grave{E}}$ & 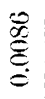 & $\begin{array}{l}\stackrel{0}{\circ} \\
\stackrel{8}{0}\end{array}$ & $\stackrel{\infty}{\stackrel{0}{3}}$ & $\stackrel{\infty}{\stackrel{\infty}{\Xi}}$ & 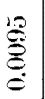 & 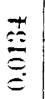 & $\stackrel{\varrho}{\varrho}$ & $\stackrel{8}{8}$ & $\begin{array}{l}0 \\
0 \\
0 \\
0 \\
0\end{array}$ & 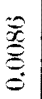 & 总 & है. & 影 & 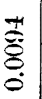 & 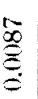 & $\begin{array}{l}\stackrel{s}{\check{E}} \\
\stackrel{\Xi}{\Xi}\end{array}$ \\
\hline & I.inks & $\begin{array}{l}\infty \\
\stackrel{\tilde{\Xi}}{0} \\
\stackrel{\circ}{\circ}\end{array}$ & 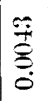 & 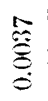 & 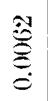 & 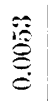 & 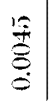 & $\begin{array}{l}\stackrel{\infty}{\stackrel{\Xi}{\Xi}} \\
\stackrel{\Xi}{\Xi}\end{array}$ & 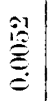 & 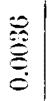 & 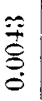 & 产 & $\begin{array}{l}0 \\
\stackrel{1}{0} \\
\stackrel{8}{0}\end{array}$ & 売 & 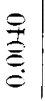 & 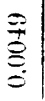 & 突 & 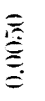 \\
\hline & Rechts & $\stackrel{\text { Pे }}{\stackrel{8}{8}}$ & 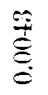 & 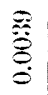 & $\stackrel{\leftrightarrow}{\circ}$ & $\stackrel{20}{\stackrel{0}{0}}$ & 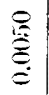 & $\begin{array}{l}\stackrel{0}{\mathscr{\delta}} \\
\stackrel{\delta}{=}\end{array}$ & 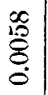 & $\begin{array}{l}\overrightarrow{\tilde{g}} \\
\stackrel{8}{8} \\
\stackrel{0}{0}\end{array}$ & 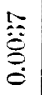 & 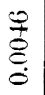 & 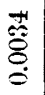 & 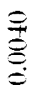 & $\begin{array}{l}\stackrel{1}{\dddot{0}} \\
\stackrel{\tilde{\delta}}{0} \\
\stackrel{0}{0}\end{array}$ & $\begin{array}{l}\hat{i} \\
\stackrel{1}{8} \\
\stackrel{8}{0}\end{array}$ & 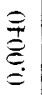 & 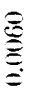 \\
\hline \multirow{4}{*}{ 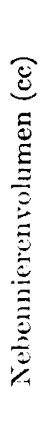 } & $\begin{array}{l}\% \text { d. Körper- } \\
\text { gewichts }\end{array}$ & $\stackrel{\stackrel{9}{\risingdotseq}}{\stackrel{9}{=}}$ & 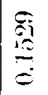 & 菜 & 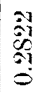 & 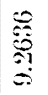 & 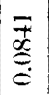 & 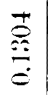 & $\frac{\widetilde{F}}{\mathrm{~F} !}$ & $\begin{array}{l}\mathfrak{\delta} \\
0 \\
0 \\
0\end{array}$ & $\stackrel{\infty}{\Xi}$ & $\frac{\overline{8}}{\stackrel{8}{0}}$ & $\begin{array}{l}\stackrel{\infty}{0} \\
\stackrel{0}{0}\end{array}$ & 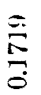 & $\begin{array}{l}\stackrel{5}{\leftrightarrows} \\
\stackrel{5}{5}\end{array}$ & $\begin{array}{l}\frac{O}{\Xi} \\
\stackrel{2}{\Xi}\end{array}$ & 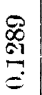 & 彦 \\
\hline & Beide & $\underset{1}{x-1}$ & 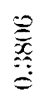 & $\frac{0}{x}$ & 赵 & 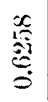 & 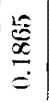 & 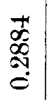 & 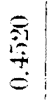 & $\begin{array}{l}\mathscr{\mathscr { B }} \\
0 \\
\stackrel{0}{=}\end{array}$ & 吝 & $\begin{array}{c}\stackrel{0}{N} \\
\frac{1}{5}\end{array}$ & 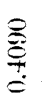 & 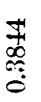 & 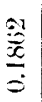 & 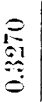 & 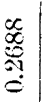 & 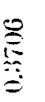 \\
\hline & Links & $\stackrel{x}{*}$ & $\hat{\tilde{\theta}}$ & $\hat{\sigma}$ & $\hat{⿳ 亠 幺 幺: ~}$ & 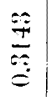 & 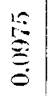 & 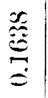 & 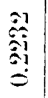 & 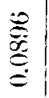 & 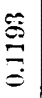 & $\begin{array}{l}\stackrel{8}{\circ} \\
\stackrel{2}{\circ} \\
\end{array}$ & 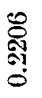 & 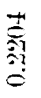 & 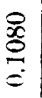 & $\begin{array}{l}\widetilde{S} \\
\stackrel{大}{E}\end{array}$ & 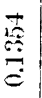 & 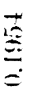 \\
\hline & Rechts & $\underset{\mathbb{C}}{\mathbb{C}}$ & $\stackrel{s}{i}$ & $\frac{\tilde{\sigma j}}{\frac{\sigma}{\delta}}$ & $\begin{array}{l}\mathscr{0} \\
\stackrel{0}{0 ! !} \\
0 \\
0\end{array}$ & 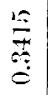 & 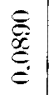 & $\begin{array}{l}0 \\
\stackrel{7}{0} \\
\stackrel{0}{0}\end{array}$ & 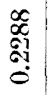 & 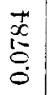 & 荵 & $\begin{array}{l}\mathscr{D} \\
\dot{0}=0 \\
0 \\
0 \\
0 \\
0\end{array}$ & $\begin{array}{l}\stackrel{D}{\infty} \\
\stackrel{\infty}{0} \\
\stackrel{0}{0}\end{array}$ & $\begin{array}{l}\text { 웜 } \\
\stackrel{0}{0}\end{array}$ & $\begin{array}{l}\hat{0} \\
\stackrel{0}{0} \\
0 \\
0 \\
0\end{array}$ & 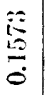 & 䓂 & $\frac{0}{i}$ \\
\hline \multicolumn{2}{|c|}{$\begin{array}{c}\text { Körpergewicht } \\
\text { (g) }\end{array}$} & $\therefore$ & $\widehat{x}$ & $\check{\Xi}$ & 埩 & $\overrightarrow{5}$ & 紧 & 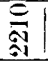 & $\begin{array}{l}15 \\
\frac{\pi}{3}\end{array}$ & $\overbrace{\substack{0\\
}}^{\infty}$ & $\underset{\bar{N}}{\Xi}$ & 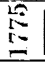 & 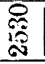 & 㯊 & $\stackrel{8}{8}$ & $\frac{\sqrt[n]{0}}{2}$ & $\begin{array}{l}\infty \\
\mathbb{N} \\
\end{array}$ & $\frac{8}{81}$ \\
\hline \multicolumn{2}{|r|}{ Geschlecht } & to & to & ot & ot & ot & or & a & њ & to & to & ot & at & ot & $\leftrightarrow$ & + & $\infty$ & or \\
\hline \multicolumn{2}{|r|}{ Nummer } & $\cong$ & $\neg$ & $\stackrel{s}{\sim}$ & $\because \hat{i}$ & 12 & 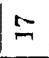 & $\stackrel{\infty}{\infty}$ & กิ & $\overline{3}$ & $\hat{\hat{i}}$ & $\cong \hat{i}$ & ลิ & 额 & $\hat{\Delta}$ & \multicolumn{3}{|c|}{$\begin{array}{l}\text { Durchsch- } \\
\text { nittswert }\end{array}$} \\
\hline
\end{tabular}


Quantitative Studie des Nebennierenmarks und -rinde beim Kaninchen.

und nur in einem Fall ist das Mark beiderseits gleich gross. Die Frequenz der Überwiegung des Marks in der Seite, wo die Nebenniere selbst grösser ist, ist $71.4 \%$. Da die linke Nebenniere in der Regel grösser als die rechte ist, ist das Mark gewöhnlich links grösser, was in 8 Fällen beobachtet wurde, dagegen war in 5 Fällen das Verhältnis umgekehrt und in einem Fall das Mark beiderseits gleich gross. Die Frequenz von $\mathrm{L} \geqslant \mathrm{R}$ ist $64.2 \%$ und die von $\mathrm{R}>\mathrm{L}, 35.8 \%$. Der Prozentsatz von $\mathrm{L}>\mathrm{R}$, sowie auch von $\mathrm{R}>\mathrm{L}$ ist resp. $26.6 \%$ (Maximum 58.3\%, Minimum 3.0\%), 13.1\% (Maximum 22.6\%, Minimum 5.4\%); also der Überwiegungsprozentsatz von $\mathrm{L}>\mathrm{R}$ ist grösser als der von $\mathrm{R}>\mathrm{L}$. Auch nach Bager (17) ist das Mark in 58 Fällen links grösser, in 38 umgekehrt und in 4 Fällen beiderseits gleich. Castaldi (22) beim Meerschweinchen und Akiyama (28) bei der Ratte beobachteten auch die gleiche Tatsache. Das Markvolumen, das Volumenprozent des Markes der beiden Nebennieren zum Organvolumen und der Rindenmarkindex sind in Tabelle VI angegeben, also durchschnittlich $0.0043 \mathrm{cc}, 3.16 \%$, 0.336 resp., was dem Wert vou Bager fast gleich ist. Nach Bager ist die Markmenge im Vergleich zur Nebenniere selbst beim Neugeborenen am grössten $(20.8 \%)$, beim 12 Monate alten Tier am kleinsten $(1.8 \%)$ und dann vermehrt sie sich allmählich bis zu etwa $3 \%$.

b) Rinde.

Die Rinde ist auffallend gross im Verhältnis zum Mark und nimmt durchschnittlich $96.84 \%$ des ganzen Volumen des Organs ein.

Nach Elliot ist die bedeutende Entwicklung der Rinde den Säugetieren eigentümlich und das relative Rindenvolumen zum Organvolumen ist beim Meerschweinchen am grössten (98.4\%), dann folgen das Kaninchen $(97.5 \%)$, die Ratte $(95.2 \%)$, die Katze $(94.5 \%)$, der Hund $(83.3 \%)$ und das Huhn $(50 \%)$.

Da die Rinde so gross im Verhältnis zum Mark ist, so sind ihre Verhätnisse zum Mark und zum Körpergewicht fast gleich wie die der ganzen Nebenniere zu den beiden letzteren.

Rindenmark-Index zeigt eine grosse Schwankung wegen der individuellen Verschiedenheit der Rindenmenge (durchschnittlich 0.0336).

Das Rindenvolumen, absolut und pro kg Körpergewicht gerechnet, die Rindenmenge im Verhältnis zum ganzen Organ und der Rindenmarkindex sind in Tabelle VI angegeben.

\section{Resultate.}

Aus der vorliegenden quantitativen Studie der Nebemniere beim 
erwachsenen Kaninchen lassen sich die Hauptresultate folgendermassen zusammenfassen.

1) Das Nebennierengewicht ist nicht immer proportional zum Körpergewicht und individuell sehr verschieden. Das absolute Gewicht beträgt durchschnittlich $0.436 \mathrm{~g}$, das relative Gewicht pro Kilogram Körpergewicht $0.1982 \mathrm{~g}{ }^{*}$ und das spezifische Gewicht 1.0371. Der Durchschnittswert der ersteren beiden Gewichte ist beim Weibchen grösser als beim Männchen.

2) Die linke Nebenniere ist in der Regel grösser als die rechte, die Frequenz von $L \geqslant R$ ist $77.8 \%$ und die von $R>L 22.2 \%$.

Der Überwiegungsprozentsatz des linken Nebennierengewichtes ist durchschnittlich $14.7 \%$ und grösser als der der rechten (4.0\%).

3) Die Markmenge ist nicht immer proportional zu der Rindengrösse und dem Körpergewicht. Die individuelle Schwankung des Markvolumens ist viel geringer als die des Rinden- und Nebennierenvolumens; so ist die Grösse der Nebenniere hauptsächlich von der der Rindengrösse abhängig.

Das Mark nimmt durchschnittlich $3.16 \%$ des ganzen Volumens der Nebenniere ein. Die absolute Menge des Markes beträgt durchschnittlich $0.0094 \mathrm{cc}$, das relative Tolumen pro Kilogramm Körpergewicht durchschnittlich 0.0043 cc.

4) Das Mark ist grösser in der Seite, welche die grössere Nebenniere trägt, dessen Frequenz durchschnittlich 71.4\%. Da die linke Nebenniere in der Regel grösser als die rechte ist, so ist das Mark auch gewöhnlich links grösser als rechts, dessen Frequenz $(L \supseteq R) 64.2 \%$ beträgt. Der Überwiegungsprozentsatz des linken Markvolumens beträgt durchschnittlich $26.6 \%$ und der des rechten $22.6 \%$.

5) Der Nebemniere-Mark-Index ist umgekehrt proportional gross zu der Rindengrösse. Daher je grösser die Nebenniere ist, desto relativ kleineres Mark besitzt sie.

6) Die Rinde ist sehr gross im Vergleich zum Mark und nimmt durchschnittlich $96.84 \%$ des ganzen Tolumens der Nebenniere ein, wodurch die Verhältnisse des Rindenvolumens zum Narkvolumen und zum Körpergewicht fast gleich den des Nebennierenvolumens zu den beiden letzteren sind.

Die Rindengrösse ist individuell sehr verschieden und in der Regel auf der linken Seite grösser als auf der rechten.

Zum Schluss möchte ich Herrn Professor K. Okajima für die freundliche Leitung bei dieser Arbeit meinen herzlichen Dank aussprechen. 
Quantitative Studie des Nebennierenmarks und -rinde beim Kaninchen.

\section{Literatur.}

1) Akiyama, S., Quantitative Studie der Nebenniere bei Ratte. (japanisch). Kaibogaku Zasshi. Bd. 1. 1928.

2) Bager, B., Bildrag till binjurarnas åldersanatomi hos kaninen. Upsala Läkareförenings Förhandlingar. Ny. följd. Bd. 23. 1917.

3) Castaldi, I.., Accresimento delle sostanze corticale e midollare della glandola surrenale e loro rapporti volumetrici. Arch. Fisol. Vol. 20. 1922.

4) Donaldson, J. C., The relative volumes of the cortex and medulla of the adrenal gland in the albino rat. Amer. Journ. Anat. Vol. 25. 1919.

5) Dostoiewsky, A., Fin Beitrag zur mikroskopischen Anatomie der Nebenieren bei Säugetieren. Arch: f. mikr. Anat. Bd. 27. 1886.

6) Elliot, 'T. R. and Tucket I., Cortex and medulla in the suprarenal glands. Journ. of Physiol. Bd. 34. 1906.

7) Gerhardt, U., Das Kaninchen. Keipzig 1909.

8) Hammer, J. A., Beiträge zur Konstitutionsanatomie VII. Methode, die Menge des Marks, der Rinde und der Rindenzonen, sowie die Menge und Verteilung der Lipoide der menschlichen Nebenniere zahlenmässig festzustellen. Zeitschr. mikr.-anat. Forschung. Bd. 1. 1924.

9) Hammar, J. A., A plea for systematic research work in the anatomy, normal and morbid, of the endocrine system. Endocrinology. Vol. 4. 1920.

10) Hett, J., Neue Untersuchungen über die Nebenniere. Verh. d. Anat. Ges. 1926.

11) - Beobachtung an der Nebenniere der Maus. II. Geschlechtsunterschiede im gegenseitigen Mengenverhältnis von Rinde und Mark bei wachsenden Tieren. Zeitsch. f. mikr.-anat. Forschung. Bd. 13. 1928.

12) Jackson, C. M., The postnatal development of the suprarenal gland and the effects of inanition upon its growth and structure in the albino rat. Amer. Journ. Anat. Vol. 25. 1919.

13) Kojima, J., On the compensatory hypertrophy of the suprarenal gland in rabbits and the relation between the survival duration after double suprarenalectomy and the accessory cortical tissue. Tohoku Journ. Experi. Med. Vol. 13. 1929.

14) Kolmer, W., Zur vergleichenden Histologie, Zytologie und Entwicklungsgeschichte der Säugernebenniere. Arch. f. mikr. Anat. Bd. 91. 1918.

15) Stilling, H., Zur Anatomie der Nebennieren. Arch. f. mikr. Anat. Bd. 52. 1898.

16) Stölzner, H., Der Einfluss der Fixierung auf das Volumen der Organe. Zeitsch. f. wiss. Mikr. Bd. 23.1906.

17) Tsukamoto, T., Entwicklungsstudie des normalen Kaninchens, besonders über das Knochensystem (japanisch). Mitteil. aus dem Pathol. Inst. der Niigata Med. Univ, 1928. 